\title{
Symmetric Image Registration
}

\author{
Peter Rogelj, Stanislav Kovačič \\ Faculty of Electrical Engineering, University of Ljubljana, Slovenia
}

\begin{abstract}
A quality of image match is usually estimated by measuring image similarity. Unfortunately, similarity measures assess only such transformations that change appearance of the deformed image, and in the case of non-rigid registration the results of the similarity measurement depend on the registration direction. This asymmetric relation leads to registration inconsistency and reduces the quality of registration. In this work we propose a symmetric registration approach, which improves the registration by measuring similarity in both registration directions. The solution presented in this paper is based on the interaction of both images involved in the registration process. Images interact with forces, which are according to the Newton's action-reaction law forming a symmetric relationship. These forces may transform both of the images, although in our implementation one of the images remains fixed. The experiments performed to demonstrate the advantages of the symmetric registration approach involve registration of simple objects, recovering synthetic deformation, and interpatient registration of real images of head. The results show improvements of registration consistency and also indicate the improvement of registration correctness.
\end{abstract}

Keywords: Non-rigid registration, symmetric registration, consistency, similarity

\section{INTRODUCTION}

The aim of image registration is to find a mapping between two imaged anatomies. As each anatomical point in the first anatomy is expected to have exactly one homologous poin in the second anatomy, correspondence mapping is expected to be one-to-one. Nevertheless, the majority of non-rigid registration systems do not provide such symmetric behavior, and registration results differ according to the registration direction. This inconsistency indicates a limited registration correctness, which is difficult to measure directly. ${ }^{1,2}$

One solution to this problem was proposed by Christensen and Johnson. ${ }^{3}$ Their consistent image registration solves the problem by jointly registering images in both registration directions and linking both processes with an additional consistency constraint. In this paper we propose an alternative approach which does not directly force the consistency, but improves the registration stage that is the most responsible for occurrence of inconsistency, i.e. measurement of image match quality.

We believe that consistency can be achieved by treating both images in the same manner, such that they both tend to improve their matching. Consequently, the quality of match is not estimated only by similarity of source image according to the target, but also by measuring similarity in the opposite direction. This improves match quality estimation, which thus becomes symmetric and registration more consistent.

Figure 1 illustrates the asymmetry of similarity measures. The transformation $\mathbf{T}$ improves the match of image $A$ to image $B$, but consists only of such transformation components that do not change the appearance of image $A$. This improvement in the quality of match cannot be detected by measuring the similarity $S(\mathbf{T} A, B)$. However, the same match as obtained by transforming image $A$ using transformation $\mathbf{T}$ can also be obtained by transforming image $B$ using the inverse transformation $\mathbf{T}^{-1}$. This inverse transformation transforms image $B$ into image $\mathbf{T}^{-1} B$, such that the appearance of image $B$ changes as well. Consequently, by measuring similarity $S\left(A, \mathbf{T}^{-1} B\right)$, the improvement of match can be detected. This illustration shows that measuring the similarity in different registration directions may detect different transformation components, which explains why registration based on such bi-directional measurements should provide better registration results.

Corresponding author:

E-mail: peter.rogelj@fe.uni-lj.si

Address: Faculty of Electrical Engineering, University of Ljubljana, Tržaška 25, 1000 Ljubljana, Slovenia 


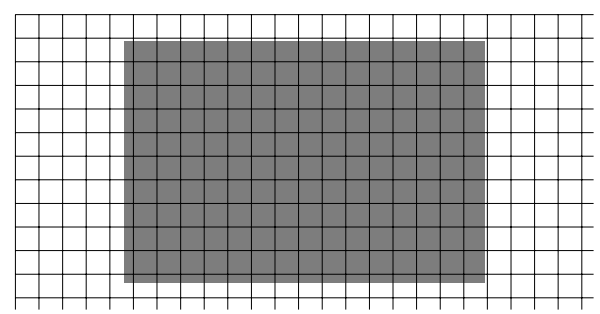

$A$

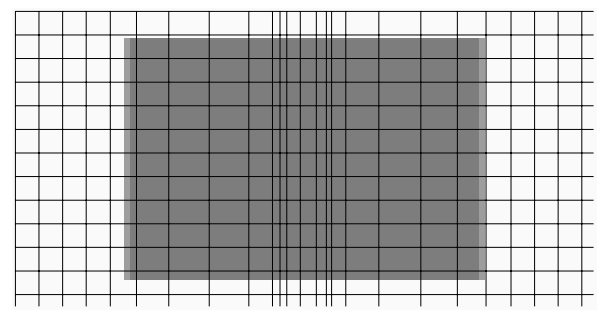

$\mathbf{T} A$

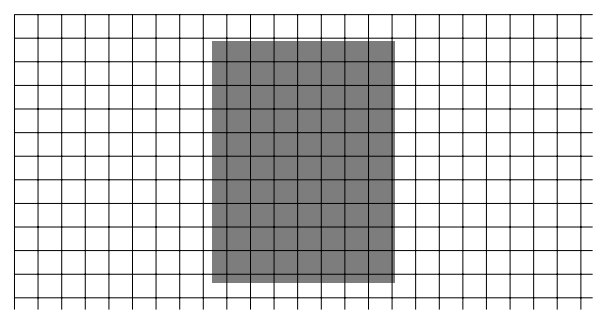

$B$

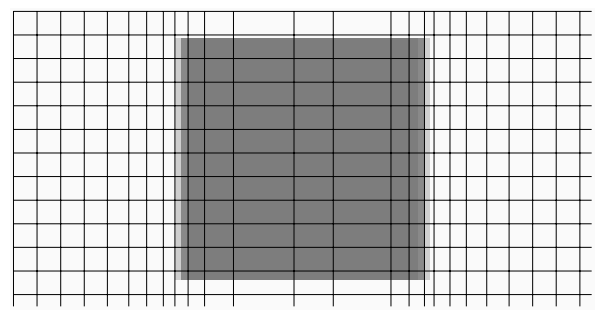

$\mathbf{T}^{-1} B$

Figure 1. Illustration of similarity measure asymmetry. Measurement of similarity in different registration directions may assess different transformation components. Similarity $S(\mathbf{T} A, B)$ cannot detect the change in image correspondence $(S(\mathbf{T} A, B)=S(A, B)=0.75)$, while similarity in the other registration direction can $\left(S\left(A, \mathbf{T}^{-1} B\right)=0.85\right)$.

Computation of inverse transformation is difficult to implement due to the discrete nature of image data, and it is also very time consuming, which makes it less suitable for iterative optimization procedures. Instead we propose a symmetric registration approach, which does not require computation of transformation inverses at all. It provides the required symmetry by allowing interaction between the images.

\section{SYMMETRIC IMAGE REGISTRATION}

The symmetric registration approach treats both images involved in the registration process equally. Both of the images share the same coordinate system and can overlap. Both of the images may be modelled by spatial deformation models, such that both of them may get transformed (they may move and/or deform). Finally, the most distinctive feature of symmetric registration approach is interaction between the images. Images interact through forces, which appear between the images, such that they tend to match similar parts of the other image. The result of the interaction is transformation of the images that puts the whole system into the equilibrium state of minimal energy. Note that in accordance with the Newton's third law of motion, forces on one image reflect in opposing forces on the other image, which forms the basis for symmetry required for obtaining the registration consistency.

If a widely used gradient descent optimization algorithm is used to find an equilibrium state, forces are defined as a gradient of estimated match quality $Q$. Note that $Q$ is an ideal measure, which is by the definition of registration related to the distances between corresponding points in the imaged anatomies. As the point correspondences are not known (that is why registration is performed), quality of match is estimated by measuring image similarity $S$. The forces that appear on image $A$ in order to match it with image $B$ are therefore the following:

$$
\mathbf{F}_{A}(\mathbf{x})=\frac{\partial S\left(\mathbf{T}_{A} A, B\right)}{\partial \mathbf{T}_{A}}
$$

In order to enable correction of very localized image differences, external forces must be estimated for correspondingly small image regions. In the extreme case these regions cover only individual image voxels. Such a situation simplifies the computation of forces, as transformation of such small image region consists only of 
translation $\mathbf{T}_{A}(\mathbf{x})$, which moves point (voxel) $A(\mathbf{x})$ from original position $\mathbf{x}$ to a new position $\mathbf{x}+\mathbf{T}_{A}(\mathbf{x})$. Here, it gets matched with a point $B\left(\mathbf{x}+\mathbf{T}_{A}(\mathbf{x})\right)$ of the other image. In this case the Eq. (1) can be rewritten in the following way:

$$
\mathbf{F}_{A}(\mathbf{x})=\frac{\partial S\left(A(\mathbf{x}), B\left(\mathbf{x}+\mathbf{T}_{A}(\mathbf{x})\right)\right)}{\partial \mathbf{T}_{A}(\mathbf{x})} .
$$

In addition to image $A$, image $B$ also tends to improve the matching. The forces $\mathbf{F}_{B}$ that are exerted on image $B$ in order to improve matching with $A$ can be calculated using the same principle as described earlier for image $A$ :

$$
\mathbf{F}_{B}(\mathbf{x})=\frac{\partial S\left(A, \mathbf{T}_{B} B\right)}{\partial \mathbf{T}_{B}}=\frac{\partial S\left(A\left(\mathbf{x}+\mathbf{T}_{B}(\mathbf{x})\right), B(\mathbf{x})\right)}{\partial \mathbf{T}_{B}(\mathbf{x})}
$$

The obtained forces, $\mathbf{F}_{A}$ and $\mathbf{F}_{B}$, which we call forward forces, are not symmetric. They are exerted on different images and estimated in the opposite registration direction, which due to the asymmetry of similarity measures reflects in differences in their size and orientation. For example, forces $\mathbf{F}_{A}$ can differ from zero only at points with nonzero gradient of image $B$, while forces $\mathbf{F}_{B}$ differ form zero at points with nonzero gradient of image $A$, see Figure 2. That asymmetry is a source of inconsistency in the case of conventional non-rigid registration approach.

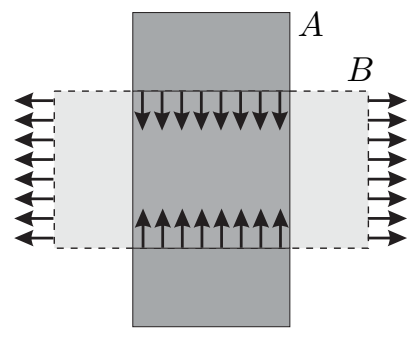

$\mathbf{F}_{A}$

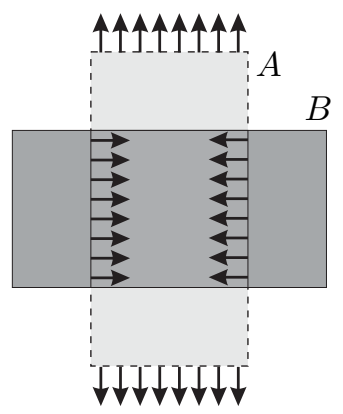

$\mathbf{F}_{B}$

Figure 2. Illustration of forces $\mathbf{F}_{A}$ acting on image $A$ and $\mathbf{F}_{B}$ acting on image $B$ when matching two rectangles. Note the asymmetry of the forces with respect to the registration direction.

In the case of symmetric image registration this is not the end of the story yet. In accordance with the Newton's third law of motion, each force exerted on one of the images reflects to another force, which applies to the other image and is equal in size and opposite in direction. Thus, forces $\mathbf{F}_{A}$ reflect to $\mathbf{F}_{A}^{\prime}$ such that $\mathbf{F}_{A}^{\prime}(\mathbf{x})=-\mathbf{F}_{A}(\mathbf{x})$, and forces $\mathbf{F}_{B}$ reflect to $\mathbf{F}_{B}^{\prime}$, such that $\mathbf{F}_{B}^{\prime}(\mathbf{x})=-\mathbf{F}_{B}(\mathbf{x})$. The new forces are called reverse forces and re illustrated in Figure 3. The resultant forces that exert on image $A$ are

$$
\mathbf{F}^{A}(\mathbf{x})=\mathbf{F}_{A}(\mathbf{x})-\mathbf{F}_{B}(\mathbf{x})
$$

and likewise the resultant forces on image $B$ are

$$
\mathbf{F}^{B}(\mathbf{x})=\mathbf{F}_{B}(\mathbf{x})-\mathbf{F}_{A}(\mathbf{x}) .
$$

For illustration of resultant forces $\mathbf{F}^{A}$ and $\mathbf{F}^{B}$ see Figure 4 .

Non-rigid image registration is usually used to transform one of the images, while the other image should remain untransformed. In this case images must be modelled by two different spatial deformation models. The reference image $A$, which should not get transformed, must be modelled as a rigid body and anchored to the coordinate system, while the source image $B$ must be modelled using suitable deformable model. Although the reference image is fixed, it does contribute to matching. The forces $\mathbf{F}_{A}$ still exist although they do not change configuration of image $A$. However, they still have influence to the source image $B$, i.e. forces $\mathbf{F}^{\prime}{ }_{A}$, still contribute to matching and improve the registration. 

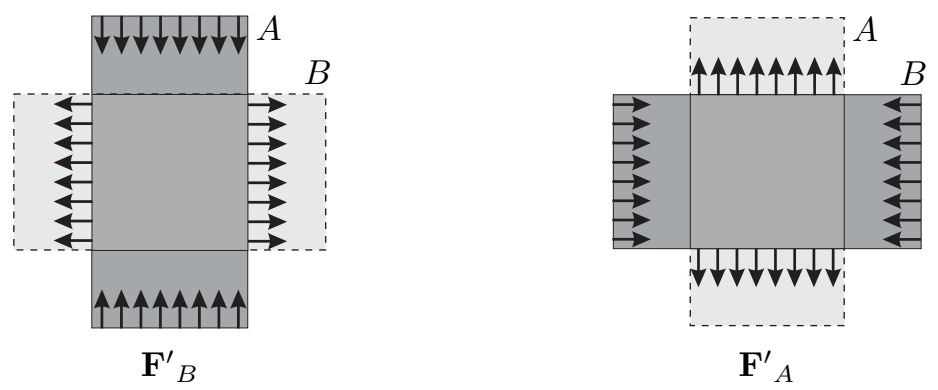

Figure 3. Illustration of reverse forces $\mathbf{F}_{B}^{\prime}$ acting on image $A$ and $\mathbf{F}^{\prime}{ }_{A}$ acting on image $B$ when matching two rectangles.

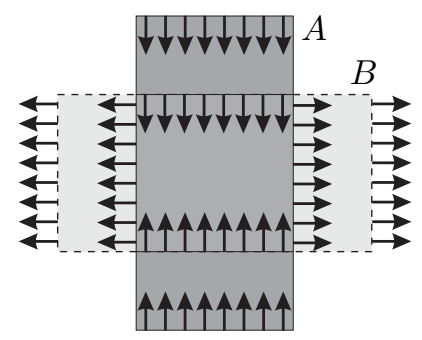

$\mathbf{F}^{A}$

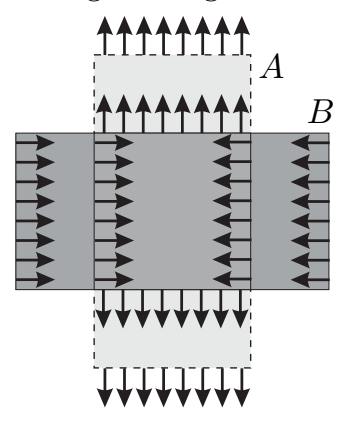

$\mathbf{F}^{B}$

Figure 4. Illustration of forces $\mathbf{F}^{A}$ acting on image $A$ and $\mathbf{F}^{B}$ acting on image $B$ when matching of two rectangles. Note the symmetry $\mathbf{F}^{A}=-\mathbf{F}^{B}$, which is required for obtaining the registration consistency.

\subsection{Implementation details}

Forces are estimated for each individual voxel of image $B$. For this purpose we use point similarity measures, ${ }^{4}$ which are capable of estimating the similarity for individual image point pair $\left(A\left(\mathbf{x}_{1}\right), B\left(\mathbf{x}_{2}\right)\right)$. Using this similarity measures, we estimate each force from points, which correspond to voxel displacements $\mathbf{T}(\mathbf{x})=$ $[+\varepsilon, 0,-\varepsilon]^{3}$ and form a $3 \times 3 \times 3$ point similarity window, see Figure 5. Similarities for points that are not positioned on the image grid are interpolated from neighboring grid points. Further estimation of forces follows Bajcsy and Kovačič. ${ }^{5}$

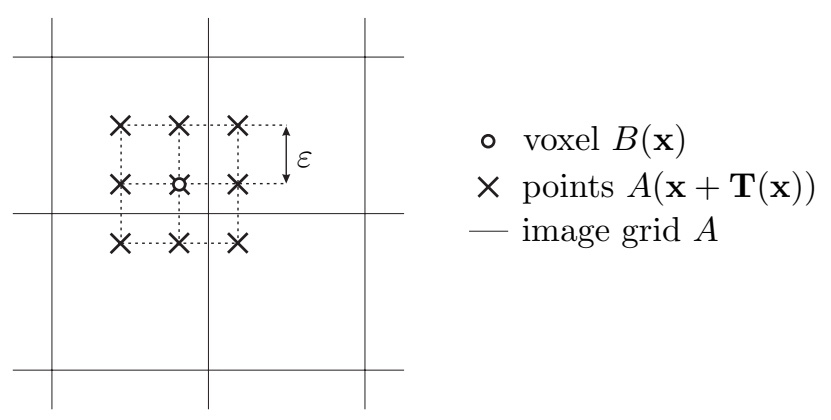

Figure 5: Points used for estimation of forward forces $\mathbf{F}_{B}$.

The estimation of reverse forces $\mathbf{F}^{\prime}{ }_{A}$ is more complex than estimation of forward forces $\mathbf{F}_{B}$, because the mapping of world coordinates into image coordinates is not directly applicable when image is deformed. Thus, to avoid the need for such mapping we estimate $\mathbf{F}_{A}$ according to the local coordinate system of (deformed) 
image $B$ and corresponding coordinates $\mathbf{x}_{B}$.

$$
\mathbf{F}_{A}\left(\mathbf{x}_{B}\right)=\frac{\partial S\left(A\left(\mathbf{x}_{B}\right), B\left(\mathbf{x}_{B}+\mathbf{T}_{A}\left(\mathbf{x}_{B}\right)\right)\right)}{\partial \mathbf{T}_{A}\left(\mathbf{x}_{B}\right)}
$$

After the forces are estimated according to the coordinate system of image $B$, they must also be defined according to the world coordinate system. The local mapping between the world coordinate system and image coordinate system is defined by Jacobian matrix $J$, see Figure 6 , such that

$$
\mathbf{F}_{A}(\mathbf{x})=J^{-1} \mathbf{F}_{A}\left(\mathbf{x}_{B}\right) .
$$

When both sets of forces, $\mathbf{F}_{B}$ and $\mathbf{F}_{A}$, are obtained, the resultant forces that deform the source image $B$ are calculated using Eq. (5).
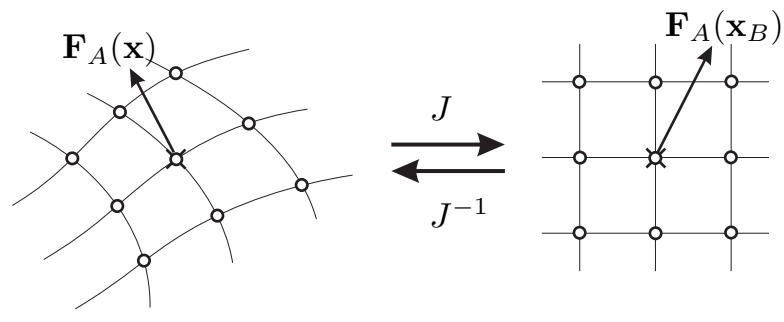

○ voxels $B\left(\mathbf{x}_{B}+\mathbf{T}\left(\mathbf{x}_{B}\right)\right)$

$\times$ point $A(\mathbf{x})$

— image grid $B$

Figure 6: Local transformation between space and image coordinate system by using a Jacobian matrix $J$.

\section{RESULTS}

Three sets of experiments were performed to demonstrate the symmetric registration approach and compare it with mono-directional approaches. The experiments involve registration of simple objects, recovering of synthetic deformations, and interpatient registration of real images of head.

Each pair of images $(A, B)$ used in the experiments was registered using the three different force estimation approaches, and in both registration directions. In total six transformations were obtained for each image pair. Registration of image $B$ to image $A$ results in transformations $\mathbf{T}_{F}$ for standard registration with forward forces $\mathbf{F}_{B}, \mathbf{T}_{R}$ for registration based on reverse forces $\mathbf{F}_{A}^{\prime}$, and $\mathbf{T}_{S}$ for symmetric registration approach driven by forces $\mathbf{F}^{B}$. When registration is performed in the opposite direction, registering image $A$ to image $B$, transformations $\mathbf{T}_{F}^{\prime}, \mathbf{T}_{R}^{\prime}$ and $\mathbf{T}_{S}^{\prime}$ are obtained, see Figure 7 .

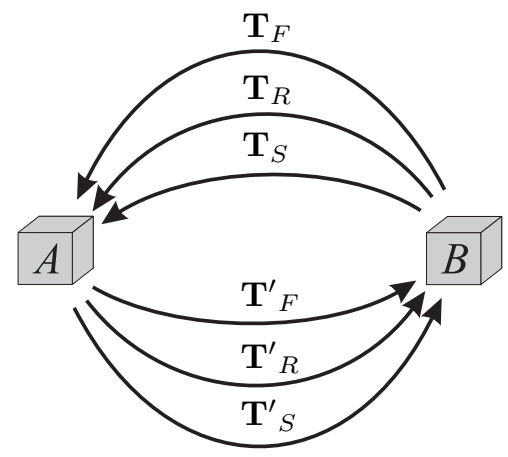

Figure 7. Transformations obtained by registering images $A$ and $B$, with respect to the method used for estimating forces (forward, reverse and symmetric) and registration direction. 
All the experiments were based on multi-modal point similarity measure using similarity function:

$$
S(\mathbf{i})=\log \frac{p(\mathbf{i})^{2}}{p\left(i_{A}\right) \cdot p\left(i_{B}\right)},
$$

where $\mathbf{i}=\left(i_{A}, i_{B}\right)$ is an intensity pair of compared points $A\left(\mathbf{x}_{1}\right)$ and $B\left(\mathbf{x}_{2}\right)$. Joint distribution $p(\mathbf{i})$ and marginal distributions $p\left(i_{A}\right)$ and $p\left(i_{B}\right)$ are estimated from the images that are being registered. The registration was regularized using a Gaussian spatial deformation model. ${ }^{1}$

Three different methods were used to assess the registration quality. First, when the correct transformation $T_{0}$ was known, the registration quality was measured directly by computing RMS displacement error $E_{R M S}$,

$$
E_{R M S}(\mathbf{T})=\sqrt{\frac{1}{N} \sum_{\mathbf{x}}\left(\mathbf{T}(\mathbf{x})-\mathbf{T}_{0}(\mathbf{x})\right)^{2}} .
$$

Here $N$ is the number of image voxels. The second method measures the registration consistency by computing a RMS deviation of point correspondences defined by transformations $\mathbf{T}$ and $\mathbf{T}^{\prime}$, which are obtained by registering the same images using the same method but in different registration directions.

$$
C_{R M S}\left(\mathbf{T}, \mathbf{T}^{\prime}\right)=\sqrt{\frac{1}{N} \sum_{\mathbf{x}}\left(\mathbf{T}(\mathbf{x})-\mathbf{T}^{\prime-1}(\mathbf{x})\right)^{2}}
$$

$\mathbf{T}^{\prime-1}$ denotes the inverse of transformation $\mathbf{T}^{\prime}$, such that it obtains the same image correspondence in the other registration direction. The last method used for assessing the registration quality was measuring of image similarity. This method is less appropriate ${ }^{1}$ as it can only judge transformation components that change the image appearance. For our experiments we used measure $S_{M A D}$ (mean absolute intensity difference) or correlation ratio $S_{C C} \cdot{ }^{6}$

\subsection{Registration of simple objects}

Two images of simple objects were generated, a rectangular prism for image $A$ and a sphere for image $B$. Central image slices are shown in Figure 8.

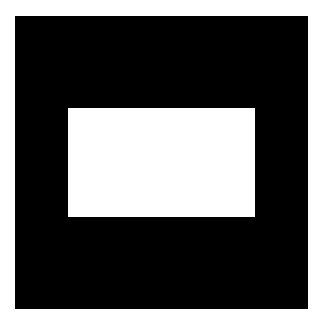

$A$

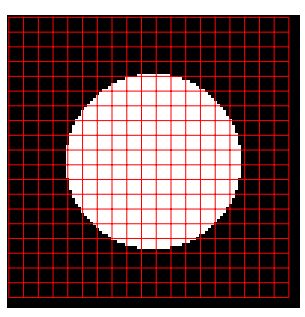

B

Figure 8. The central slices of the simple 3D images, representing a rectangular prism (image $A$ ) and a sphere (image $B)$.

After registering the images with all three registration approaches in both directions, the results were compared by measuring consistency $C_{R M S}$ and similarity of registered images $S_{C C}(A, \mathbf{T} B)$. As the ideal transformation $\mathbf{T}_{0}$ was not known in this case, the registration error $E_{R M S}$ could not be obtained. The results are shown in Figure 9. Although all three resulting images look very similar, the consistency measure $C_{R M S}$ indicates the advantage of the symmetric approach. The results of the other two methods are worse, especially when using only the reverse forces (transformation $T_{R}$ ). However, different conclusions could be drawn when observing the image similarity. Here, the method based on the reverse forces gives the best result, better than the symmetric approach. To find out which of the approaches is more correct we performed the second experiment, based on recovering synthetic deformation. 


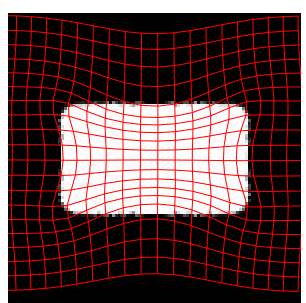

$\mathbf{T}_{F} B$

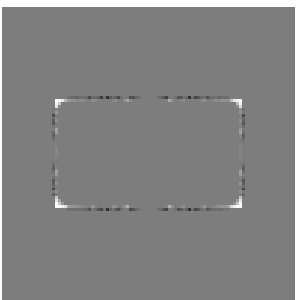

$A-\mathbf{T}_{F} B$

$C_{R M S}=1.349$

$S_{C C}=0.968$

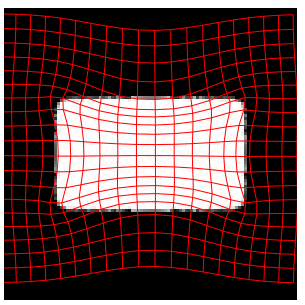

$\mathbf{T}_{R} B$

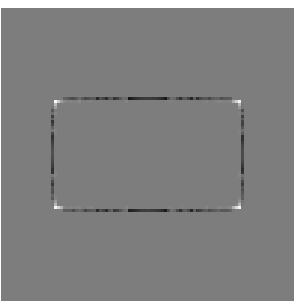

$A-\mathbf{T}_{R} B$

$C_{R M S}=1.658$

$S_{C C}=0.984$

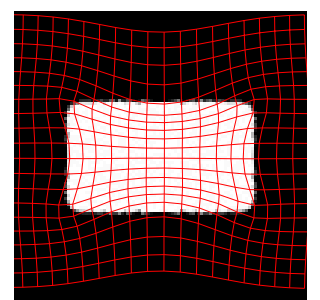

$\mathbf{T}_{S} B$

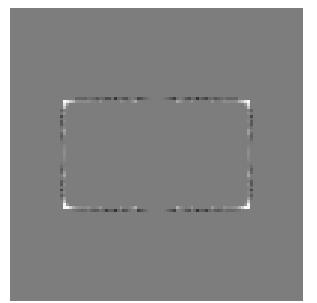

$A-\mathbf{T}_{S} B$

$C_{R M S}=0.893$

$S_{C C}=0.976$

Figure 9. Results of registering the simple image pair using different methods for estimating external forces. The first row represents the resulting images $\mathbf{T} B$, and the second row shows the difference to the reference image $A$. Below the images, consistency results $C_{R M S}$ and final image similarities $S_{M A D}$ are shown for each of the methods.

\subsection{Recovering synthetic deformations}

In this experiment Brainweb ${ }^{7}$ simulated images of human head were used. Firstly, the original MRI-T1 image was used for $A$ and its synthetically deformed version for $B$. Secondly, we performed a multi-modality registration by using MRI-PD image as a reference $A$, while $B$ remained the same synthetically deformed MRI-T1 image, see Figure 10.

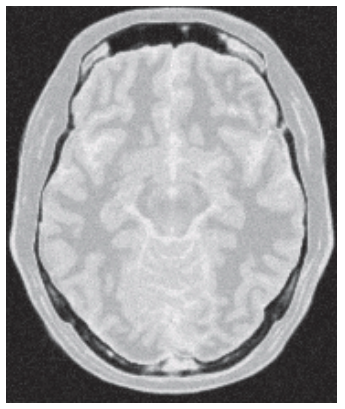

MRI-PD

$A$ (multi-modality)

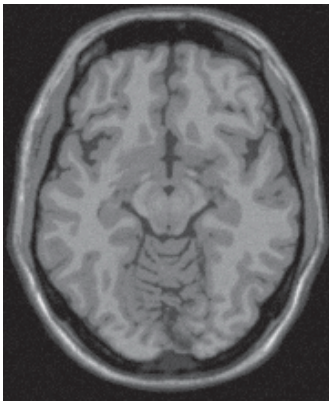

MRI-T1

$A$ (mono-modality)

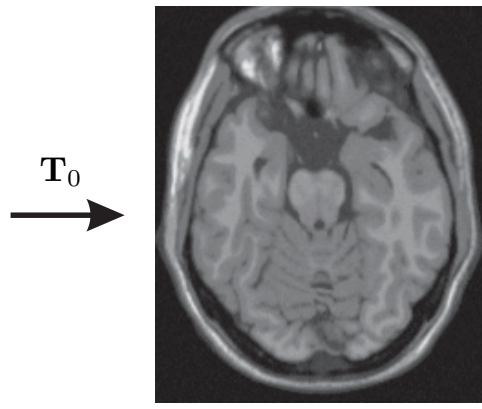

MRI-T1

$B$

Figure 10. Images used for the experiment based on recovering synthetic deformations. Image $A$ was an original untransformed MRI-T1 image (mono-modality registration) or MRI-PD image (multi-modality registration). Image $B$ was in all the cases generated by deforming the original MRI-T1 image.

Five different synthetic deformations $\mathbf{T}_{0}$ were used, each of them generated as a sum of five three-dimensional 
Gaussian functions with randomly selected parameters, where the amplitude was in the range of 0 to $26 \mathrm{~mm}$, and standard deviation was in the range of 5 to $50 \mathrm{~mm}$.

The synthetically generated transformation $\mathbf{T}_{0}$ enabled the evaluation of registration correctness $E_{R M S}$, the consistency $C_{R M S}$ and image similarity $S_{M A D}$. Because the original images MRI-T1 and MRI-PD were in register, measure $S_{M A D}$ and original MRI-T1 image were used also for evaluation of the multi-modal registration results $(\mathbf{T} B)$. The results are tabulated in Table 1 . In all the cases the symmetric approach performed best with regard to the registration correctness and registration consistency, and in general it was also best according to the image similarity. The results were obtained for mono-modality as well as for multi-modality registration.

Table 1: Results of recovering the synthetic deformations.

\begin{tabular}{|c|c||c|c|c||c|c|c|}
\hline Experiment & \multicolumn{1}{|c||}{ Method } & \multicolumn{2}{c||}{ Mono-modality registration } & \multicolumn{3}{c|}{ Multi-modality registration } \\
& & $E_{R M S}$ & $C_{R M S}$ & $S_{M A D}$ & $E_{R M S}$ & $C_{R M S}$ & $S_{M A D}$ \\
\hline \hline \multirow{3}{*}{$\mathbf{T}_{0}^{(1)}$} & $\mathbf{T}_{F}$ & 1.267 & 0.548 & 5.080 & 1.302 & 0.854 & 5.365 \\
& $\mathbf{T}_{R}$ & 1.382 & 1.570 & 4.869 & 1.876 & 2.050 & 5.225 \\
& $\mathbf{T}_{S}$ & 1.052 & 0.322 & 4.674 & 1.108 & 0.400 & 4.941 \\
\hline \multirow{3}{*}{$\mathbf{T}_{0}^{(2)}$} & $\mathbf{T}_{F}$ & 1.520 & 0.908 & 4.038 & 1.241 & 1.020 & 3.823 \\
& $\mathbf{T}_{R}$ & 1.358 & 1.885 & 3.785 & 1.791 & 2.086 & 3.903 \\
& $\mathbf{T}_{S}$ & 1.242 & 0.356 & 3.537 & 1.093 & 0.326 & 3.541 \\
\hline \multirow{3}{*}{$\mathbf{T}_{0}^{(3)}$} & $\mathbf{T}_{F}$ & 1.413 & 0.785 & 4.542 & 1.716 & 0.819 & 4.698 \\
& $\mathbf{T}_{R}$ & 1.225 & 2.066 & 4.030 & 1.760 & 2.200 & 4.307 \\
& $\mathbf{T}_{S}$ & 0.981 & 0.768 & 4.104 & 1.040 & 0.672 & 4.037 \\
\hline \multirow{2}{*}{$\mathbf{T}_{0}^{(4)}$} & $\mathbf{T}_{F}$ & 1.043 & 0.305 & 3.545 & 1.166 & 0.565 & 3.733 \\
& $\mathbf{T}_{R}$ & 1.233 & 1.509 & 3.833 & 1.801 & 2.001 & 4.106 \\
& $\mathbf{T}_{S}$ & 0.919 & 0.192 & 3.341 & 1.037 & 0.255 & 3.650 \\
\hline \multirow{2}{*}{$\mathbf{T}_{0}^{(5)}$} & $\mathbf{T}_{F}$ & 1.580 & 1.005 & 4.544 & 1.661 & 1.103 & 4.747 \\
& $\mathbf{T}_{R}$ & 1.501 & 1.663 & 4.036 & 1.970 & 2.203 & 4.312 \\
& $\mathbf{T}_{S}$ & 1.425 & 0.491 & 4.058 & 1.521 & 0.509 & 4.228 \\
\hline \hline \multirow{2}{*}{ average } & $\mathbf{T}_{F}$ & 1.578 & 0.916 & 4.585 & 1.417 & 0.872 & 4.473 \\
& $\mathbf{T}_{R}$ & 1.489 & 1.731 & 4.165 & 1.840 & 2.108 & 4.371 \\
& $\mathbf{T}_{S}$ & 1.330 & 0.510 & 4.091 & 1.160 & 0.433 & 4.080 \\
\hline
\end{tabular}

\subsection{Registration of real interpatient data}

In the last experiment real MRI-T1 images were used. Specifically, six images were used, such that one of them always served as image $A$ and the other five images were used as $B$. Thus, altogether there were five image pairs, each of them required six transformations (three methods in both directions). Example images are shown in Figure 11.

The registration results were evaluated by measuring the consistency $C_{R M S}$ and image similarity $S_{C C}$. Because the images differ in brightness and contrast, the correlation measure $S_{C C}$ was used. The results are tabulated in Table 2. One can observe that consistency of symmetric registration is in all cases much better than the consistency of the other two methods.

\subsection{Discussion}

All the results show that symmetric registration approach is more consistent than standard registration approaches. Furthermore the results of recovering synthetic deformations show that symmetric registration also improves the registration correctness. However, the improvement of consistency is larger than the improvement of registration correctness. This is mainly due to the fact that similarity measures do not directly assess the quality of image match. The symmetric approach does overcome the problem of similarity measure asymmetry and in that aspect make similarity measuring consistent, but gradients of similarity and external forces still 


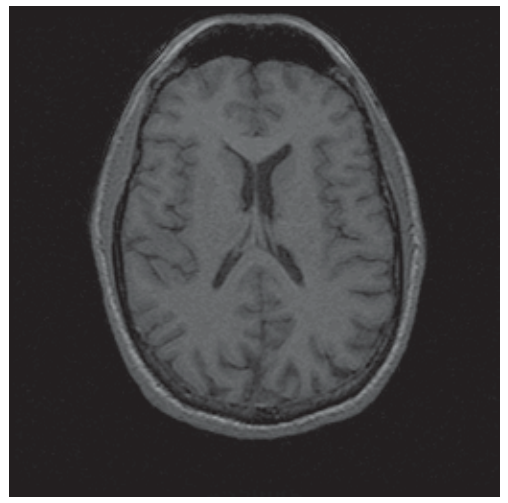

$A$

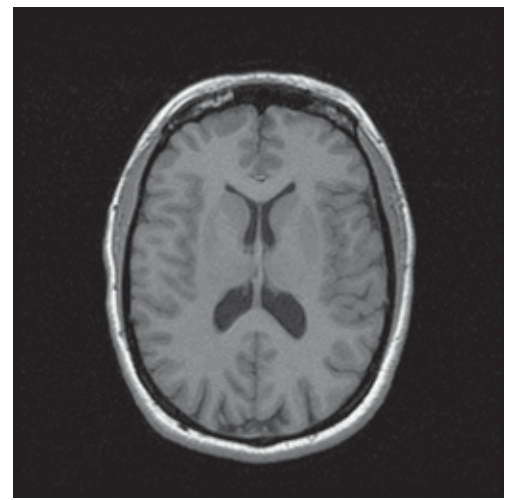

$B$

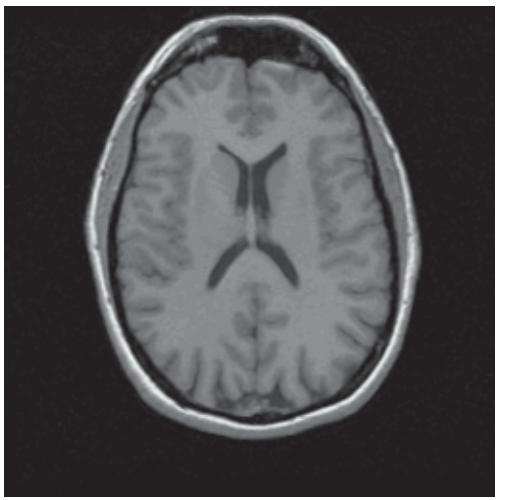

$\mathbf{T} B$

Figure 11. Interpatient registration of real MRI-T1 head images. Image $A$ is a reference used for registering image $B$, and $\mathbf{T} B$ is the registered image.

Table 2: Results of interpatient registration of real images of head.

\begin{tabular}{|c|c||c|c|}
\hline Experiment & Method & $C_{R M S}$ & $S_{C C}$ \\
\hline \hline \multirow{3}{*}{ MRI01 } & $\mathbf{T}_{F}$ & 3.090 & 0.788 \\
& $\mathbf{T}_{R}$ & 4.859 & 0.839 \\
& $\mathbf{T}_{S}$ & 1.384 & 0.818 \\
\hline \multirow{3}{*}{ MRI02 } & $\mathbf{T}_{F}$ & 3.145 & 0.851 \\
& $\mathbf{T}_{R}$ & 5.136 & 0.878 \\
& $\mathbf{T}_{S}$ & 0.963 & 0.875 \\
\hline \multirow{3}{*}{ MRI03 } & $\mathbf{T}_{F}$ & 3.155 & 0.859 \\
& $\mathbf{T}_{R}$ & 4.142 & 0.864 \\
& $\mathbf{T}_{S}$ & 1.062 & 0.870 \\
\hline \multirow{3}{*}{ MRI04 } & $\mathbf{T}_{F}$ & 3.410 & 0.874 \\
& $\mathbf{T}_{R}$ & 5.323 & 0.880 \\
& $\mathbf{T}_{S}$ & 1.023 & 0.883 \\
\hline \multirow{3}{*}{ MRI05 } & $\mathbf{T}_{F}$ & 2.951 & 0.863 \\
& $\mathbf{T}_{R}$ & 4.747 & 0.881 \\
& $\mathbf{T}_{S}$ & 0.911 & 0.880 \\
\hline \hline & $\mathbf{T}_{F}$ & 3.150 & 0.847 \\
& $\mathbf{T}_{R}$ & 4.841 & 0.868 \\
& $\mathbf{T}_{S}$ & 1.068 & 0.865 \\
\hline
\end{tabular}

appear only at the edges of imaged objects. This renders the exact registration of homogeneous image regions impossible. To improve the registration correctness of these regions a suitable spatial deformation model must be used. This model should allow only such deformations that can in reality appear inside these regions.

The results also show that the final image appearance becomes most similar to the reference image when only the reverse forces are used. This is because the reverse forces always act on the edges of objects in the image that is being deformed. As such all the external energy is used exclusively for transformations that improve appearance of the final deformed image, and not for other transformation components, which are required to obtain consistent or better match of homogeneous image regions.

On the other hand, the forward forces appear at the edges of objects in the reference image, which is not necessarily only at the edges present in the image, which is being registered. Actually, during the registration process these forces are applied to different points of this image, which contributes to the registration consistency.

The consistency of the symmetric registration approach is still not as good as one would expect. Here, we 
have to emphasize that differences between results obtained in different registration directions are also caused by spatial deformation model, or to be more specific, by using different initial image configuration as the undeformed state of anatomy. ${ }^{8}$ Because properties of real tissues are not symmetric, two transformations that are inverses of each other require different deformation energy. Therefore, more correct registration results can be obtained when the correct undeformed configuration of anatomy is presumed. This is a source of differences, which are normally understood as inconsistency, although they may also be desired.

\section{CONCLUSION}

This paper presents new aspects of the registration process, which result in the proposed symmetric registration approach. Its main contribution is in the solution to the similarity measure asymmetry problem, which was the main source of registration inconsistency.

Three different experiments were performed to demonstrate the symmetric registration approach and compare it with two standard mono-directional approaches. The results show that the symmetric registration approach does not improve only the consistency but also improves the registration correctness.

The symmetric approach in general allows both images to be modelled by suitable deformable model and thus both of them could actually deform. Such approach could better suit to certain registration tasks and provide better registration results. In addition to this generalization, it would also be possible to use more than two images, which would interact with forces at the same time.

Finally, note that the registration process is completely symmetric only if both of the images are modelled using the same spatial deformation model. However, when this is not the case, the remaining asymmetry can not be always understood as inconsistency, because it may also be desired.

\section{ACKNOWLEDGMENTS}

The authors gratefully acknowledge the support of the Slovenian Ministry of Education, Science and Sport (Research program 1538-517).

\section{REFERENCES}

1. P. Rogelj, S. Kovačič, and J. Gee, "Validation of a nonrigid registration algorithm for multimodal data," in Medical Imaging 2002: Image Processing, M. Sonka and F. J.M., eds., Proc. SPIE 4684, pp. 299-307, SPIE Press, Bellingham, WA, 2002.

2. R. Woods, "Validation of registration accuracy," in Handbook of Medical Imaging Processing and Analysis, I. Bankman, ed., pp. 491-497, Academic Press, 1999.

3. G. Christensen and H. Johnson, "Consistent image registration," IEEE Transactions on Medical Imaging 20, pp. 568-582, July 2001.

4. P. Rogelj and S. Kovačič, "Similarity measures for non-rigid registration," in Medical Imaging 2001: Image Processing, M. Sonka and K. Hanson, eds., Proc. SPIE 4322, pp. 569-578, SPIE Press, Bellingham, WA, 2001.

5. R. Bajcsy and S. Kovačič, "Multiresolution elastic matching," Computer Vision, Graphics and Image Processing 46, pp. 1-21, April 1989.

6. D. L. G. Hill and D. J. Hawkes, "Across-modality registration using intensity-based cost functions," in Handbook of Medical Image Processing, I. Bankman, ed., pp. 537-553, Academic Press, 1999.

7. R.-S. Kwan, A. Evans, and G. B. Pike, "An extensible MRI simulator for post-processing evaluation," in Visualization in Biomedical Computing (VBC'96), Lecture Notes in Computer Science 1131, pp. 135-140, Springer-Verlag, May 1996.

8. P. Cachier and D. Rey, "Symmetrization of the non-rigid registration problem using invertion-invariant energies : Application to multiple sclerosis," in Proceedings of the 3rd International Conference on Medical Image Computing and Computer-Assisted Intervention - MICCAI 2000, S. L. Delp, A. M. DiGioia, and B. Jaramaz, eds., Lecture Notes in Computer Science 1935, pp. 472-481, Springer, 2000. 